\title{
Neuropathic pain: transcranial electric motor cortex stimulation using high frequency random noise. Case report of a novel treatment
}

\author{
This article was published in the following Dove Press journal: \\ Journal of Pain Research \\ 22 June 2013 \\ Number of times this article has been viewed
}

\author{
Per A Alm \\ Karolina Dreimanis \\ Department of Neuroscience, Uppsala \\ University, Uppsala, Sweden
}

Objectives: Electric motor cortex stimulation has been reported to be effective for many cases of neuropathic pain, in the form of epidural stimulation or transcranial direct current stimulation (tDCS). A novel technique is transcranial random noise stimulation (tRNS), which increases the cortical excitability irrespective of the orientation of the current. The aim of this study was to investigate the effect of tRNS on neuropathic pain in a small number of subjects, and in a case study explore the effects of different stimulation parameters and the long-term stability of treatment effects.

Methods: The study was divided into three phases: (1) a double-blind crossover study, with four subjects; (2) a double-blind extended case study with one responder; and (3) open continued treatment. The motor cortex stimulation consisted of alternating current random noise (100-600 Hz), varying from 0.5 to 10 minutes and from 50 to $1500 \mu \mathrm{A}$, at intervals ranging from daily to fortnightly.

Results: One out of four participants showed a strong positive effect (also compared with direct-current-sham, $P=0.006)$. Unexpectedly, this effect was shown to occur also for very weak $(100 \mu \mathrm{A}, P=0.048)$ and brief $(0.5$ minutes, $P=0.028)$ stimulation. The effect was largest during the first month, but remained at a highly motivating level for the patient after 6 months.

Discussion: The study suggests that tRNS may be an effective treatment for some cases of neuropathic pain. An important result was the indication that even low levels of stimulation may have substantial effects.

Keywords: neuropathic pain, central pain, transcranial direct current stimulation, motor cortex stimulation, random noise stimulation

\section{Introduction}

\section{Central neuropathic pain}

Central neuropathic pain is associated with lesions of the brain or spinal cord. A range of proposals regarding the pathological mechanisms has been put forward. In a recent review of research it was argued that current evidence points towards reverberation in a loop between the primary somatosensory cortex (S1) and the sensory thalamus, resulting in over excitation of the cortical region. ${ }^{1}$ A related proposal is the concept of "thalamocortical dysrhythmia," by Llinás. ${ }^{2}$ According to this model, deafferentation results in a functionally deactivated area of the $\mathrm{S} 1$, which may show pathological slow wave oscillations with the thalamus $(\leq 9 \mathrm{~Hz}) \cdot{ }^{3-5}$ In this model the actual sensation of pain is the result of an "edge effect," ie, hyperactivation of the border of the deactivated cortical region, with spontaneous gamma oscillation in cortical columns which have lost the normal GABAergic collateral inhibition. ${ }^{4,6}$ The amplitude of gamma oscillations
Correspondence: Per A Alm Biomedical Center, Uppsala University, Box 593, SE-75I 24 Uppsala, Sweden

Tel +46 I8 47| 4743

$\mathrm{Fax}+4618511540$

Email per.alm@neuro.uu.se 
$(60-95 \mathrm{~Hz})$ in the $\mathrm{S} 1$ has been reported to be closely related to the subjective experience of nociceptive pain. ${ }^{7}$

\section{Neuromodulation as treatment of neuropathic pain}

Chronic neuropathic pain often shows insufficient response to pharmacological treatments. An alternative approach is electrical neuromodulation, in various forms. Transcutaneous electrical nerve stimulation (TENS) and spinal cord stimulation are two methods which, however, result in long-term relief only in a few cases of central neuropathic pain. ${ }^{1}$ Deep brain stimulation (DBS) of thalamic nuclei has become relatively widely used, but the success for treating central pain has not fulfilled the initial hopes. ${ }^{1,8}$ The effects appear to depend on excitation of neurons, with increased blood flow in the thalamus and in the S1. ${ }^{1}$ Yet another method is cortical stimulation, which will be discussed in more detail below.

\section{Cortical stimulation, $\mathrm{MI}$ and $\mathrm{SI}$}

\section{Epidural motor cortex stimulation (EMCS)}

In EMCS the relevant area of the primary motor cortex (M1) is stimulated by electrodes which typically are placed outside the dura. Compared with DBS this method is less invasive and poses fewer risks. Reports of long-term efficacy have been relatively encouraging. In a study of 32 patients with neuropathic pain, the average pain ratings on the visual analog scale (VAS) was reduced from 86 before surgery to 40 about 2 years later. ${ }^{10}$ Six of these patients showed a progressive loss of effect during the first months, but for five of them a repositioning of the electrodes with better somatotopical match restored the effect. In a study of 15 patients with peripheral neuropathic pain, the follow-up at 9-12 months showed a 58\%-95\% reduction of VAS scores in eight patients, a $31 \%-45 \%$ reduction in two, and a $0 \%-11 \%$ reduction in five. ${ }^{11}$ EMCS for trigeminal neuropathic pain has been claimed to show clinically significant long-term results in more than $70 \%$ of the patients. ${ }^{8}$

Typical stimulation parameters for EMCS are $40 \mathrm{~Hz}$ of $60 \mu$ s direct current (DC) pulses at $2 \mathrm{~V}^{11}$ which is far below the threshold for muscle contraction. ${ }^{12}$ It has been proposed that the optimal placement can be determined by finding the optimal location for anodal elicitation of a motor response in the relevant body part. This location is used for the cathode in pain treatment, with the anode above or just posterior to the central sulcus. ${ }^{13,14}$ Other authors give less weight to the exact placement of the electrodes, and in some studies the electrodes have been placed also above S1. ${ }^{1}$
Repetitive transcranial magnetic stimulation (rTMS) rTMS induces electrical currents in the cortex. The effect depends on the frequency of the pulses, with high frequency $\left(\geq 5 \mathrm{~Hz}\right.$ ) rTMS over M1 resulting in cortical excitation ${ }^{15}$ and pain reducing effects. ${ }^{16-18}$ The typical coil is focal, with about $1 \mathrm{~cm}$ core stimulation. This allows very specific stimulation, but also requires exact navigation. The typical duration of the pain reduction has been reported as between 5 minutes and 8 days, but some studies suggest longer effect (about 2 weeks) after several consecutive sessions. ${ }^{1}$ There seems to be a paucity of reports of long-term treatment using rTMS. It has been shown that reduction of pain after a single session of rTMS predicts a positive response to epidural stimulation, whereas lack of response to rTMS has no value as a criterion for exclusion from surgery. ${ }^{19}$

\section{Transcranial DC stimulation (tDCS)}

tDCS is a comparatively recent noninvasive method, in which electrodes are applied to the surface of the head. The constant $\mathrm{DC}(\leq 2 \mathrm{~mA})$ is virtually unnoticeable. It has been shown that the anode and the cathode have opposite effects on the excitability of the underlying cortex, with increase under the anode and decrease under the cathode. ${ }^{20}$

Several double-blind studies of chronic pain have shown an effect of anodal tDCS over M1, ${ }^{21-24}$ typically with 20 minutes of stimulation during 5 consecutive days. At a group level these studies have shown a decrease of VAS ratings down to between $42 \%$ and $87 \%$ compared with baseline, with relatively long duration. In ten patients with neuropathic pain due to multiple sclerosis the average improvement was $63 \%$, which largely outlasted the follow-up period of 4 weeks. ${ }^{23}$ Among 12 patients with neuropathic pain of mixed etiology eight showed at least $30 \%$ reduction of pain, and about half of the effect remained 4 weeks later. ${ }^{22}$ Studies of neuropathic pain in spinal cord injury have shown mixed results, with large differences between individuals. ${ }^{21,24}$

M1 tDCS may be a non-invasive alternative to epidural stimulation in some cases. One difference between the methods is that tDCS is less focal, so that the electric field will affect a large portion of the M1 and S1.

\section{Transcranial random noise stimulation (tRNS)}

tRNS is a novel method, using alternating current with randomly varying frequencies, resulting in a "white noise" waveform. It has been shown that 10 minutes of $1 \mathrm{~mA}$ transcranial stimulation with $100-640 \mathrm{~Hz}$ random noise results in increased excitability of M1 in healthy persons, lasting more than 1 hour. ${ }^{25,26}$ In contrast to tDCS, both electrodes have an 
activating effect, and it is assumed that neurons will become activated regardless of orientation. ${ }^{25} \mathrm{~A}$ putative mechanism is that the alternating electric field results in repeated opening and closing of voltage-gated sodium channels, resulting in multiple ionic influxes and a shift of membrane potential towards the action potential. ${ }^{25,27,28}$ The safety of tRNS has been evaluated by measurements of neuron-specific enolase (NSE, a sensitive marker for neuronal damage, with increase in epilepsy), and electroencephalography (EEG). No effects on NSE or EEG have been found..$^{25} \mathrm{tRNS}$ is well suited for blinded studies since the stimulation as such typically is not noticed, and the threshold for cutaneous sensation is higher for tRNS than for tDCS. ${ }^{29}$ In a study of visual discrimination, tRNS over visual cortex improved performance, compared with tDCS and sham. ${ }^{30}$

To the best of our knowledge a recent case study on major depression $^{31}$ is the first report of the use of tRNS as treatment for a pathological condition. The patient had shown poor response to pharmacological treatment and psychotherapy. In this non-blind trial tRNS was compared with tDCS, resulting in a stronger treatment effect for tRNS. The rationale for the stimulation was to induce increased activity in the left dorsolateral prefrontal cortex.

The studies reviewed in previous sections suggest that neuromodulation for pain is related to excitation of M1 and/or the thalamus. ${ }^{1,15-18,21-24}$ Considering this background, tRNS may be a potentially effective method for treatment of neuropathic pain. Some potential strengths of tRNS are the same as for tDCS, being a method that is noninvasive, easy to use, and with the possibility that inexpensive devices for home treatment can be manufactured. In addition, if the excitatory effect of tRNS is unrelated to the orientation of the neurons in relation to the current, tRNS may have the effect of exciting a larger number of cortical neurons compared with tDCS, independent of the cortical folding, and in both hemispheres. This excitatory effect may also include the M1 of the medial wall (related to the lower extremities), and possibly also the thalamus.

The purpose of this study was to investigate the possible use of tRNS for treatment of neuropathic pain in a small number of subjects, and in the case study, explore the effects of different parameters and long-term stability.

\section{Materials and methods Study design}

The study consisted of three phases. Phase 1 was a doubleblind crossover study, in which all participants received periods of full stimulation (10 minutes) and "intended sham" (0.5 minutes, determined by the design of the stimulation device). Each period consisted of sessions during 5 consecutive days, with at least a 2-week interval between the periods. The order of sham versus active stimulation was balanced, with randomization in pairs of participants.

In phase 2 responders were offered continued stimulation. This was done in the form of a double-blind sham-controlled time-series case study, in which the principal investigator could change the settings based on the response of the participants, while the person operating the stimulator was unaware of the settings of the stimulator (the display of the device was partly masked). Phase 2 was started with a baseline period, without sessions, before the first stimulation. Phase 3 was an open trial in which the stimulation continued with the purpose of best effect.

\section{Participants}

Patients with epilepsy, cranial damage, metal implants in the head, or a cardiac pacemaker were excluded from the study. The participants in phase 1 were four males suffering from spinal cord injury and neuropathic pain, age 32 to 62 years, with mean age 44 years.

Three of these cases did not continue to phase 2 , due to lack of response. Their pain was related to spinal cord injuries 4,8 , and 20 years before the trial. One participant, age 38 , continued to phase 2 . He was suffering from neuropathic pain with allodynia in the left hand and arm, originating from a traumatic incident 5 years before the start of the trial. The trauma resulted in brain contusions and spinal cord injuries at multiple locations. Also, the motor functions of the left arm were affected, with hand dystonia and occasional twitches. In addition, he experienced relatively constant pain on the right side of the back, and paresis of the right leg. Due to the multiple lesions, the exact origin of the neuropathic pain was not clear. Before and during the trial, he was treated with $225 \mathrm{mg}$ pregabalin daily.

All treatments were given with written informed consent, and the study was approved by the Regional Research Ethics Committee.

\section{Ratings}

The level of pain was estimated using a VAS scale from 0 to 10 (where 10 meant worst possible pain) directly before and after each stimulation, and at follow-up 1 week after each period. During phase 2 and 3, the responder provided VAS ratings each morning and evening, separate for pain in the left hand/arm and for the right side of the back, and for the motor function of the right leg with paresis $(0=$ no impairment; $10=$ the worst level of paresis for you). 


\section{Stimulation}

Stimulation was delivered from a battery-driven stimulator (DC-Stimulator PLUS, The Magstim Company Limited, Whitland, Wales, UK), with saline-soaked surface sponge electrodes, $6 \times 4 \mathrm{~cm}$. One electrode was placed approximately over the hand area of the right M1, contralateral to the side of the dominating pain. The other electrode was placed approximately over the face area of M1, on the other side of the head. The other electrode was placed on the contralateral side anterior to the upper part of the ear, approximately over the face representation of M1. The full stimulation consisted of 10 minutes of high frequency bipolar Gaussian random noise $(100-600 \mathrm{~Hz})$, at the level of $1500 \mu \mathrm{A}$ with 10 seconds of fade in/out. The "intended sham" in phase 1 was determined by the built-in study-mode of the stimulator, and consisted of 0.5 minutes of $1500 \mu \mathrm{A}$ tRNS, plus 10 seconds of fade in/out. In addition, during the remainder of the time during sham stimulation, short pulses occurred for the purpose of impedance control: $110 \mu \mathrm{A}$, with pulse duration

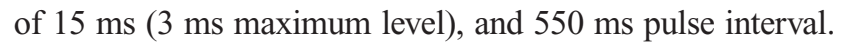
The total charge during this "intended sham" was $7 \%$ of the charge for full stimulation.

Because there were strong indications that the 0.5 minute stimulation intended as sham also had an effect on the pain, a primary aim of phase 2 was to establish a valid sham, which did not have any significant positive effects on the pain. Therefore, different levels of current, duration, and stimulation types were tested. The sham used in the statistical analyses consisted of 30 seconds of $25 \mu \mathrm{A}$ DC stimulation, in double-blind trials. Because this level of stimulation was assumed to be inactive, the polarity of the electrodes during the DC sham was not recorded.

\section{Statistics}

The time series were tested for autocorrelation using the Ljung-Box test, showing no significant autocorrelation (based on asymptotic chi-square approximation). The Ljung-Box test was applied using the IBM SPSS Statistics software (IBM Corporation, Armonk, NY, USA). Thereafter, the statistical significance of treatment effects was tested using the MannWhitney $\mathrm{U}$ test, because a normal distribution could not be assumed. These tests were performed using the software Statistica 10 (StatSoft Inc, Tulsa, OK, USA).

\section{Results}

\section{Phase I: double-blind crossover study VAS ratings}

Three of the four participants showed no systematic indications of pain reduction related to the stimulation. The fourth person showed strong positive results, however, including during the "intended sham" condition: see Figure 1. He also reported that the motor functions of the right leg had improved. The fact that this participant also responded to the "intended sham" condition is consistent with a placebo effect. However, an alternative interpretation is that the 0.5 minute stimulation was sufficient to reduce pain. This interpretation is supported to some extent by the tendency toward a doseresponse relationship: see Figure 1.

\section{Side effects}

Side effects were evaluated by means of a questionnaire after each stimulation, and by an interview before the next stimulation. Mild cutaneous sensations were reported after $15 \%$ of the sessions. Patient 4, also showing reduced pain, was the only participant reporting other side effects after the 10-minute stimulation: headache lasting 2 hours on one occasion, and mild dizziness on two occasions.

\section{Phase 2: double-blind extended time series case study}

Patient 4 was highly motivated to continue stimulation, and was started on phase 2 , consisting of 12 tRNS sessions during a 3-week period. VAS ratings morning and evening during a 9-day baseline period (no sessions) resulted in a baseline mean VAS of 8.5 (standard deviation $=1.3$ ).

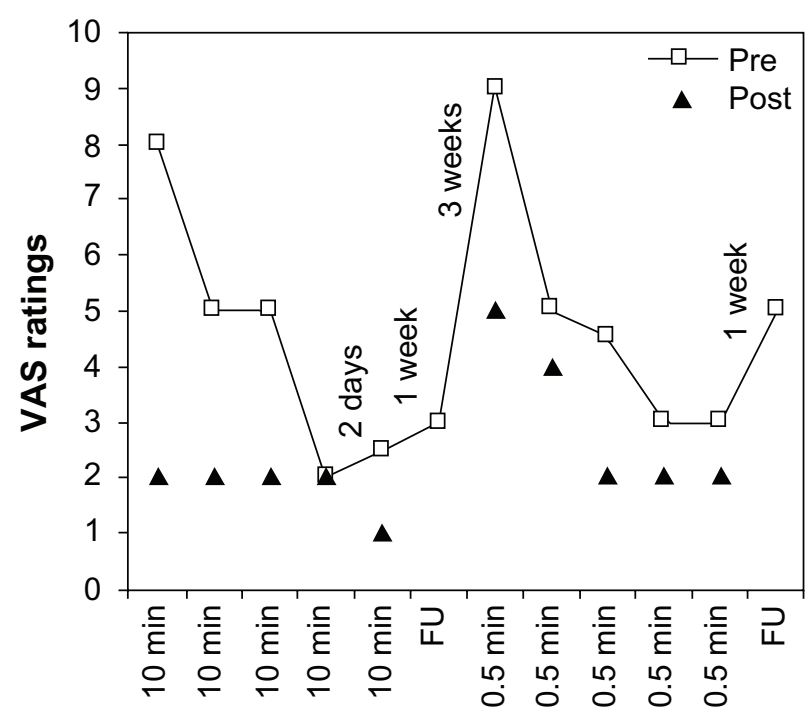

Figure I VAS ratings for patient 4 during phase I, double-blind crossover study. Notes: Two periods of five daily stimulation sessions, 10 minutes versus 0.5 minutes (intended sham), respectively. Squares indicate VAS ratings before stimulation (pre) and triangles indicate ratings directly after (post).

Abbreviations: FU, follow-up at I week; min, minute; VAS, visual analog scale. 
Table I Immediate pain-reducing effect of tRNS (varying duration and current) versus DC-sham ( $25 \mu \mathrm{A}$ for 30 seconds), in doubleblinded sessions for patient 4

\begin{tabular}{|c|c|c|c|c|c|c|c|}
\hline & \multirow[t]{2}{*}{$n$} & \multicolumn{2}{|c|}{$\begin{array}{l}\text { Mean immediate pain } \\
\text { reducing effect }\end{array}$} & \multirow[t]{2}{*}{$\begin{array}{l}\text { SD, } \\
\%\end{array}$} & \multirow[t]{2}{*}{ Range } & \multirow[t]{2}{*}{$P$} & \multirow[t]{2}{*}{$\overline{\text { Cohen's d, effect size }}$} \\
\hline & & VAS unit & $\%$ & & & & \\
\hline tRNS, all blind & 20 & 1.9 & $40 \%$ & $21 \%$ & $0 \%$ to $75 \%$ & 0.006 & 1.8 \\
\hline DC-sham & 5 & 0.1 & $2 \%$ & $22 \%$ & $-11 \%$ to $41 \%$ & & \\
\hline
\end{tabular}

Notes: Effect calculated as percentage reduction of VAS ratings for arm/hand, directly before and after stimulation. P-value from Mann-Whitney $U$ Test. Effect size was calculated as Cohen's $d$.

Abbreviations: DC, direct current stimulation; SD, standard deviation; tRNS, transcranial random noise stimulation; VAS, visual analog scale.

\section{Achieving inactive sham}

In order to verify the effect of stimulation on pain, one intention was to see if it was possible to find a level of stimulation that did not result in reduced VAS ratings. To be able to distribute the sham with very low levels of current, the display of the device was masked for the operators. However, running the device with low current proved difficult, because it sometimes interrupted stimulation due to high impedance. In addition, the VAS ratings continued to be reduced despite very low levels of tRNS. Therefore, the settings for sham were changed to $\mathrm{DC}$ at $25 \mu \mathrm{A}$ for 30 seconds. This DC-sham was used on five occasions, without indications of a treatment effect (mean immediate change of VAS ratings: -0.1 , with standard deviation $=0.8$ ).

However, during the last of these five sessions with the DC-sham, the subject reported headache and increased pain, and the session was interrupted. For the next 24 hours the pain ratings increased from the mean level of 2.6 during the previous week to about 7.7. The patient was convinced that the increase of pain was caused by the stimulation, and it was decided that the trial should change from blind to open sessions (phase 3). After one additional session of tRNS the pain ratings stabilized around 3.5. ${ }^{20}$ (Based on data from healthy individuals ${ }^{20}$ tDCS of $25 \mu \mathrm{A}$ for 30 seconds is far below the threshold for any physiological effects, and therefore the polarity of this sham stimulation was not recorded. The adverse response of the patient after the last session with DC-sham may be pure coincidence. However, it can not be excluded that a pathological unstable neural circuit can be affected by minute electrical perturbations which do not affect a healthy stable circuit. Because the polarity of the sham DC stimulation was not recorded it can not be excluded that the differences in responses to DC-sham were related to different polarity, possibly with increased pain after inhibiting cathodal stimulation. No similar sudden episodes of increased pain have been reported by the patient.)

\section{Placebo effect}

A key question is whether the electrical stimulation had any real effect on the subjective pain, or if it was just random or placebo effects. Table 1 shows a strongly significant immediate effect of blind tRNS sessions in phase 1 and 2, compared with the DC-sham.

\section{Effect of low current tRNS}

\section{Low current tRNS versus DC-sham}

The attempts to use tRNS with low current as an intended sham indicated that current from $200 \mu \mathrm{A}$ and below may show an effect on pain. Comparison of low current tRNS sessions $(\mathrm{n}=7)$ with DC-sham sessions $(\mathrm{n}=5)$ showed a significant treatment effect of low current tRNS ( $P=0.048$ for VAS improvement, Mann-Whitney U Test), with effect size Cohen's $d=1.7$. The mean current and duration for these tRNS sessions was $79 \mu \mathrm{A}$ and 6.1 minutes.

\section{Low current versus high current tRNS}

For comparison of the effect of low current tRNS (mean $100 \mu \mathrm{A})$ and high current tRNS $(1500 \mu \mathrm{A})$, the sessions were selected so they were matched for (1) length of stimulation, (2) the time-period for stimulation during the study, and (3) pre-stimulation VAS-ratings (sessions with pre-stimulation rating $<2.5$ were excluded): see Table 2 . The effects of low and high current tRNS were very similar, both regarding immediate effect and effect by the next day. In fact, the mean effects were slightly higher for the low current sessions, though this difference was not statistically significant.

\section{Effect of short duration tRNS Short duration tRNS versus DC-sham}

Figure 1 suggests that short duration tRNS (30 seconds) may have had an effect on pain for patient 4 . Short duration tRNS sessions $(n=9)$ showed a significant effect on immediate VAS improvement ( $P=0.028$, Mann-Whitney U Test) compared with DC-sham sessions $(\mathrm{n}=5)$, with effect size Cohen's $d=1.5$. 
Table 2 Mean effects of matched sessions with high versus low current tRNS (I500 $\mu \mathrm{A}$ versus on average $100 \mu \mathrm{A}$ ) for patient 4

\begin{tabular}{|c|c|c|c|c|c|c|}
\hline & \multirow[t]{2}{*}{$\mathbf{n}$} & \multicolumn{2}{|c|}{$\begin{array}{l}\text { Mean } \\
\text { immediate pain } \\
\text { reducing effect }\end{array}$} & \multirow[t]{2}{*}{ SD } & \multirow[t]{2}{*}{$\begin{array}{l}\text { Mean VAS } \\
\text { improvement } \\
\text { by next day }\end{array}$} & \multirow[t]{2}{*}{ SD } \\
\hline & & VAS unit & $\%$ & & & \\
\hline tRNS high & 10 & 1.38 & $34 \%$ & 1.0 & 0.55 & 1.0 \\
\hline tRNS low & 4 & 1.53 & $39 \%$ & 1.0 & 0.95 & 1.7 \\
\hline
\end{tabular}

Abbreviations: SD, standard deviation; tRNS, transcranial random noise stimulation; VAS, visual analog scale.

\section{Short versus long duration tRNS}

For comparison of the effect of short sessions of tRNS (30 seconds) and long sessions (10 minutes) the sessions were selected so that they were matched for (1) current level, (2) the time-period for stimulation during the study, and (3) for pre-stimulation VAS-ratings (sessions with pre-stimulation rating $<2.5$ were excluded): see Table 3 . The mean effects were higher for long tRNS, though this was not statistically significant with this sample size.

\section{All phases, including phase 3 (not blind) Effects of long-term treatment}

The main effects of tRNS on arm pain are shown in Table 4. The VAS ratings for all periods are significantly lower than baseline (Mann-Whitney $\mathrm{U}$ test, $P<0.0001$ ).

\section{Correlation between symptoms}

The VAS ratings of the pain in the arm were highly correlated with the ratings of the pain in the back and the paresis of the $\operatorname{leg}\left(P<0.01, r_{\text {back }}=0.89, r_{\text {leg }}=0.76\right)$. This provides support for the reliability of the study, and indicates that the tRNS stimulation also affected the pain in the right side of the back and the right leg paresis.

\section{Duration of after-effects}

Analysis of the after-effects during pauses indicates a linear decrease of the effect, with a total duration in the magnitude of weeks. In the beginning of the study (phase 2) the slopes were relatively steep, with around 5 points of increase in pain

Table 3 Mean effects of matched sessions with long versus short tRNS ( 10 minutes versus 30 seconds) for patient 4

\begin{tabular}{llllll}
\hline & $\mathbf{n}$ & $\begin{array}{l}\text { Mean } \\
\text { improvement }\end{array}$ & SD & $\begin{array}{l}\text { Mean VAS } \\
\text { improvement } \\
\text { by next day }\end{array}$ & SD \\
\hline tRNS 10 min & 11 & 2.21 & 1.9 & 1.28 & 1.5 \\
tRNS 0.5 min & 8 & 1.49 & 0.4 & 1.16 & 1.7 \\
\hline
\end{tabular}

Abbreviations: SD, standard deviation; tRNS, transcranial random noise stimulation; VAS, visual analog scale. per week, while the slope was flatter during phase 3, around 1 point of increase in pain per week.

\section{Discussion Main results}

As far as we know this is the first study of the effects of tRNS on neuropathic pain. The double-blind case study showed a strong positive effect on pain as well as the paresis for one of the four participants. Unexpectedly, a significant positive effect compared with the DC-sham was shown for very weak $(100 \mu \mathrm{A})$ as well as brief stimulation $(0.5$ minutes $)$. In fact, the mean effect of low current sessions was somewhat higher than the mean effect of high current sessions $(1500 \mu \mathrm{A})$, though this was not significantly different. The initial effects were large, with pain reduction from a baseline of 8.5 down to about 2 on the VAS. After about 1 month of stimulation the effects became smaller, with an average VAS score of 5.6 over 5 months. The study suggests that frequent stimulation may result in reduced long-term effect. The optimal parameters remain to be determined, and may vary between individuals.

\section{Limitations and strengths of the study}

The main limitations of the study come from the explorative ad hoc procedures in phase 2 and 3 , which were motivated by the indications in phase 1 that brief stimulation intended as a sham may also have had a significant effect for the responding case. The added blind data from phase 2 provides support for this contention, with statistically significant differences both for brief and weak tRNS stimulation compared with the DC sham. There is a need for replication of this finding, but it suggests that studies using tRNS for neuropathic pain ideally should use sham stimulation consisting of no current at all. tRNS stimulation typically does not result in cutaneous sensations, ${ }^{29}$ making blinding with zero-current sham a realistic alternative.

Another limitation of the study comes from the varying stimulation intervals during phase 2 , which may result in varying cumulative effects of stimulation. However, a prerequisite for varying cumulative effects is that the stimulation actually had an effect, otherwise there will be no accumulation of effects. This means that varying cumulative effects may have influenced the exact figures in the results, but are unlikely to have resulted in false positive findings.

A major strength of the study is the long-term follow-up. As far as we know, this is the first long-term report of tRNS/ tDCS for neuropathic pain. The preliminary data from this study point to the possibility that frequent stimulation (eg, every day) may be negative for the long-term sustainability of a treatment effect. For the clinical utility of tRNS/tDCS, 
Table 4 Results for the baseline and the 3 phases: mean VAS ratings for pain in arm, mean percentage immediate improvement of pain at each session of tRNS, and effect size Cohen's $d$ compared with baseline

\begin{tabular}{|c|c|c|c|c|c|c|c|c|}
\hline \multirow[t]{2}{*}{ Period } & \multirow[t]{2}{*}{ n Days } & \multirow[t]{2}{*}{ n VAS ratings } & \multirow[t]{2}{*}{ n tRNS sessions } & \multicolumn{3}{|c|}{ Arm pain, VAS } & \multicolumn{2}{|c|}{$\begin{array}{l}\text { Immediate } \\
\text { reduction of arm } \\
\text { pain by tRNS }\end{array}$} \\
\hline & & & & Mean & SD & Cohen's d & Mean & SD \\
\hline Baseline: & 8 & 17 & - & 8.5 & 1.3 & & & \\
\hline Phase I: & 25 & 10 & 10 & 3.8 & 1.2 & 3.7 & $44 \%$ & $23 . \%$ \\
\hline Phase 2: & 21 & 47 & 12 & 3.0 & 1.1 & 4.6 & $31 \%$ & $22 \%$ \\
\hline Phase 3: & 156 & 190 & 20 & 5.6 & 1.0 & 2.5 & $18 \%$ & $11 \%$ \\
\hline
\end{tabular}

Note: Ratings before the first stimulation in each period are excluded.

Abbreviations: SD, standard deviation; tRNS, transcranial random noise stimulation; VAS, visual analog scale.

it is essential to conduct further long-term studies of optimal treatment parameters.

\section{Cellular mechanisms}

The mechanisms of voltage-gated ion channels are comparatively well understood, but the implications for cortical neuromodulation are not yet clear. It is possible that published data from in vitro studies of voltage-gated ion channels may be informative regarding the effects of different stimulation parameters, and may motivate further in vitro experiments.

\section{tRNS for neuropathic pain}

\section{Screening before surgery}

tRNS and tDCS have the potential of becoming costeffective treatment methods, with inexpensive stimulation modules for home use being programmed by the caregiver. Another possible use is single sessions of tRNS to predict the effect of epidural stimulation before surgery. Compared with rTMS $^{19}$ the stimulation of rTNS is less focal and may therefore be easier to use for prediction of the effect of surgery.

\section{Predicting the response to tRNS: duration and stability of symptoms}

In this study, only one out of four persons with neuropathic pain showed an effect during phase 1. A possible factor influencing the effect may be the time since the lesion occurred. In a previous study of tDCS for neuropathic pain after spinal cord injury, only participants with a duration shorter than 4 years showed a clear effect. ${ }^{21}$ In the present study, the cases which did not respond had durations of 4,8 , and 20 years, which might have contributed to the lack of response. It may be the case that persons with fluctuating symptoms have the best prognosis for effect, because the fluctuations indicate that the state is not fixed.

\section{tRNS versus tDCS, parameters}

It has recently been shown that anodal tDCS resulted in large positive effects on neuropathic pain due to multiple sclerosis, with long duration of the after-effect. ${ }^{23}$ It would be of interest to compare the effects of tDCS and tRNS in double-blind crossover studies. Participants may be included in such a study based on their responses to a single stimulation session, in order to avoid repeated ineffective stimulation. Furthermore, neuropathic pain is a disorder that is well suited for double-blind case studies to investigate the effects of varying stimulation parameters, for example using stimulation with different parameters once a week.

\section{tRNS for lower body pain}

Motor cortex stimulation for lower body regions poses a problem, because the cortex is hidden within the medial wall of the hemispheres. Electrodes for epidural stimulation cannot be applied close to the cortex. The orientation of the medial cortex makes it more complicated to predict the effects of tDCS. Because the effects of tRNS appear to be insensitive to the orientation of the neurons, it may well be possible to apply tRNS stimulation also for body regions represented on the medial wall.

\section{Acknowledgments}

Thanks to Karin Rudling and Fariba Rezaei Ahan for valuable support regarding facilities for the study, to Hans Arinell for statistical advice, and to Robert George Dewsnap for linguistic review.

\section{Disclosure}

The authors report no conflicts of interest in this work.

\section{References}

1. Canavero S, Bonicalzi V. Central pain syndrome: pathophysiology, diagnosis, and management. Cambridge, UK: Cambridge University Press; 2011. 
2. Llinás RR, Ribary U, Jeanmonod D, Kronberg E, Mitra PP. Thalamocortical dysrhythmia: a neurological and neuropsychiatric syndrome characterized by magnetoencephalography. Proc Natl Acad Sci U S A. 1999;96(26):15222-15227.

3. Sarnthein J, Jeanmonod D. High thalamocortical theta coherence in patients with neurogenic pain. Neuroimage. 2008;39(4):1910-1917.

4. Walton KD, Llinas RR. Central Pain as a Thalamocortical Dysrhythmia: a Thalamic Efference Disconnection? In: Kruger L, Light AR, editors. Translational Pain Research: From Mouse to Man. Boca Raton, FL: CRC Press; 2010.

5. Walton KD, Dubois M, Llinás RR. Abnormal thalamocortical activity in patients with Complex Regional Pain Syndrome (CRPS) type I. Pain. 2010;150(1):41-51.

6. Llinás R, Urbano FJ, Leznik E, Ramírez RR, van Marle HJ. Rhythmic and dysrhythmic thalamocortical dynamics: GABA systems and the edge effect. Trends Neurosci. 2005;28(6):325-333.

7. Gross J, Schnitzler A, Timmermann L, Ploner M. Gamma oscillations in human primary somatosensory cortex reflect pain perception. PLos Biol. 2007;5(5):e133.

8. Nguyen JP, Lefaucheur JP, Raoul S, Roualdes V, Péréon Y, Keravel Y. Treatment of trigeminal neuropathic pain by motor cortex stimulation. Neurochirurgie. 2009;55(2):226-230. French.

9. Rezai AR, Lozano AM, Crawley AP, et al. Thalamic stimulation and functional magnetic resonance imaging: localization of cortical and subcortical activation with implanted electrodes. Technical note. J Neurosurg. 1999;90(3):583-590.

10. Nguyen JP, Lefaucheur JP, Decq P, et al. Chronic motor cortex stimulation in the treatment of central and neuropathic pain. Correlations between clinical, electrophysiological and anatomical data. Pain. 1999;82(3):245-251.

11. Lefaucheur JP, Drouot X, Cunin P, et al. Motor cortex stimulation for the treatment of refractory peripheral neuropathic pain. Brain. 2009;132(Pt 6): $1463-1471$.

12. Lefaucheur JP, de Andrade DC. Intraoperative neurophysiologic mapping of the central cortical region for epidural electrode placement in the treatment of neuropathic pain by motor cortex stimulation. Brain Stimul. 2009;2(3):138-148.

13. Holsheimer J, Lefaucheur JP, Buitenweg JR, Goujon C, Nineb A, Nguyen JP. The role of intra-operative motor evoked potentials in the optimization of chronic cortical stimulation for the treatment of neuropathic pain. Clin Neurophysiol. 2007;118(10):2287-2296.

14. Manola L, Roelofsen BH, Holsheimer J, Marani E, Geelen J. Modelling motor cortex stimulation for chronic pain control: electrical potential field, activating functions and responses of simple nerve fibre models. Med Biol Eng Comput. 2005;43(3):335-343.

15. Fitzgerald PB, Fountain S, Daskalakis ZJ. A comprehensive review of the effects of rTMS on motor cortical excitability and inhibition. Clin Neurophysiol. 2006;117(12):2584-2596.

16. Saitoh Y, Hirayama A, Kishima H, et al. Reduction of intractable deafferentation pain due to spinal cord or peripheral lesion by high-frequency repetitive transcranial magnetic stimulation of the primary motor cortex. J Neurosurg. 2007;107(3):555-559.
17. Ahmed MA, Mohamed SA, Sayed D. Long-term antalgic effects of repetitive transcranial magnetic stimulation of motor cortex and serum beta-endorphin in patients with phantom pain. Neurol Res. 2011;33(9): 953-958.

18. Picarelli H, Teixeira MJ, de Andrade DC, et al. Repetitive transcranial magnetic stimulation is efficacious as an add-on to pharmacological therapy in complex regional pain syndrome (CRPS) type I. J Pain. 2010;11(11):1203-1210.

19. Lefaucheur JP, Ménard-Lefaucheur I, Goujon C, Keravel Y, Nguyen JP. Predictive value of rTMS in the identification of responders to epidural motor cortex stimulation therapy for pain. J Pain. 2011;12(10): 1102-1111.

20. Nitsche MA, Paulus W. Excitability changes induced in the human motor cortex by weak transcranial direct current stimulation. J Physiol. 2000;527(Pt 3):633-639.

21. Fregni F, Boggio PS, Lima MC, et al. A sham-controlled, phase II trial of transcranial direct current stimulation for the treatment of central pain in traumatic spinal cord injury. Pain. 2006;122(1-2):197-209.

22. Antal A, Terney D, Kühnl S, Paulus W. Anodal transcranial direct current stimulation of the motor cortex ameliorates chronic pain and reduces short intracortical inhibition. J Pain Symptom Manage. 2010;39(5):890-903.

23. Mori $\mathrm{F}$, Codecà $\mathrm{C}$, Kusayanagi $\mathrm{H}$, et al. Effects of anodal transcranial direct current stimulation on chronic neuropathic pain in patients with multiple sclerosis. J Pain. 2010;11(5):436-442.

24. Soler MD, Kumru H, Pelayo R, et al. Effectiveness of transcranial direct current stimulation and visual illusion on neuropathic pain in spinal cord injury. Brain. 2010;133(9):2565-2577.

25. Terney D, Chaieb L, Moliadze V, Antal A, Paulus W. Increasing human brain excitability by transcranial high-frequency random noise stimulation. J Neurosci. 2008;28(52):14147-14155.

26. Chaieb L, Paulus W, Antal A. Evaluating aftereffects of short-duration transcranial random noise stimulation on cortical excitability. Neural Plast. 2011;2011:105927.

27. Tombola F, Pathak MM, Isacoff EY. How does voltage open an ion channel? Annu Rev Cell Dev Biol. 2006;22:23-52.

28. Dryga A, Chakrabarty S, Vicatos S, Warshel A. Realistic simulation of the activation of voltage-gated ion channels. Proc Natl Acad Sci USA. 2012;109(9):3335-3340.

29. Ambrus GG, Paulus W, Antal A. Cutaneous perception thresholds of electrical stimulation methods: comparison of tDCS and tRNS. Clin Neurophysiol. 2010;121(11):1908-1914.

30. Fertonani A, Pirulli C, Miniussi C. Random noise stimulation improves neuroplasticity in perceptual learning. J Neurosci. 2011;31(43): 15416-15423.

31. Chan HN, Alonzo A, Martin DM, et al. Treatment of major depressive disorder by transcranial random noise stimulation: case report of a novel treatment. Biol Psychiatry. 2012;72(4):e9-e10.
Journal of Pain Research

\section{Publish your work in this journal}

The Journal of Pain Research is an international, peer-reviewed, open access, online journal that welcomes laboratory and clinical findings in the fields of pain research and the prevention and management of pain. Original research, reviews, symposium reports, hypothesis formation and commentaries are all considered for publication.

Submit your manuscript here: http://www.dovepress.com/journal-of-pain-research-journal

\section{Dovepress}

The manuscript management system is completely online and includes a very quick and fair peer-review system, which is all easy to use. Visit http://www.dovepress.com/testimonials.php to read real quotes from published authors. 